Check for updates

Cite this: RSC Adv., 2018, 8, 217

\title{
Synthesis of hyperbranched polyglycerols using ascorbic acid as an activator
}

\author{
Mehdi Dadkhah, ${ }^{a}$ Hamidreza Shamlooei, ${ }^{a}$ Ehsan Mohammadifar ${ }^{b}$ \\ and Mohsen Adeli (iD *a
}

In this work, low molecular weight hyperbranched polyglycerols (LMPGs) are synthesized in bulk using ascorbic acid as an activator. Different molar ratios of glycidol and ascorbic acid were mixed and stirred at ambient conditions and LMPGs with different molecular weights, degree of branching and relative abundance of the structural units were obtained. According to spectroscopy data, ascorbic acid was not incorporated in the structure of the polymer and plays the role of activating agent in the polymerization process. In this synthetic protocol, no other chemical reagents and organic solvents were used. Therefore, products are free of toxic impurities and suitable for future biomedical applications.

Received 28th November 2017

Accepted 13th December 2017

DOI: 10.1039/c7ra12861d

rsc.li/rsc-advances

synthesized by biocompatible initiators and in aqueous solu-

\section{Introduction}

Hyperbranched polyglycerols (hPGs), highly functional hydrophilic aliphatic polyether polyols, have been explored and developed as one of the most promising candidates for drug delivery and other biomedical purposes. Unique physicochemical and biological properties such as water solubility, multifunctionality which provides many potential sites for the conjugation of therapeutic agents and multivalent interactions, excellent biocompatibility, moderate thermal stability, oxidation resistance, no significant effects on the conjugates and low toxicity cause high interest for their usage in biological applications. ${ }^{\mathbf{1 - 4}}$ Furthermore, because of their polyether structure and their similarity to PEG, hPGs are expected to show intrinsic ability to avoid mononuclear phagocytic system uptake. ${ }^{2,5}$ Also, the in vivo circulation half-lives of hPGs could be controlled by manipulation of their molecular weights. ${ }^{6,7}$ These advantages make the hPGs suitable alternatives to the conventional PEG for a broad range of biomedical applications. ${ }^{8-11}$ Since the first report on the synthesis of polyglycerol by Sandler et al. in 1966, ${ }^{12}$ different methods for the preparation of linear and hyperbranched analogues of this polymer, based on anionic and cationic ring opening polymerization of glycidol, have been established. ${ }^{3,13-19}$ However, a big challenge for the preparation of polyglycerols by conventional anionic and cationic ring opening polymerization is to use toxic initiators, catalysts and organic solvents. ${ }^{20-22}$ Traces of toxic agents in the final products induce adverse effects on the biological systems and hamper the biomedical applications of polyglycerols. ${ }^{23,24}$ Recently, polyglycerols with the defined structural parameters are

${ }^{a}$ Department of Chemistry, Lorestan University, Khorramabad, Iran. E-mail: mohadeli@yahoo.com

${ }^{b}$ School of Chemistry, University College of Science, University of Tehran, Tehran, Iran tions. ${ }^{25,26}$ In these procedures, in addition to aqueous polymerization medium, nontoxic initiators reduce the health risk of the synthesized polyglycerols. Recently, in a collaborative work, we showed that citric acid is able to initiate the polymerization of glycidol and incorporate into the structure of products, leading to biocompatible and biodegradable polyglycerols. ${ }^{27}$ Herein, we show that ascorbic acid (vitamin C) is able to activate the polymerization of glycidol at ambient conditions. Structure of the obtained hyperbranched polyglycerols could be somehow tuned by manipulation the ratio of glycidol to ascorbic acid and reaction conditions.

\section{Experimental}

Glycidol (purity 96\%) and ascorbic acid L (+) were purchased from Sigma Aldrich and Merck, respectively. The dialysis membrane tubing (Biotech Cellulose Ester) (width: $31 \mathrm{~mm}$, MWCO: $2 \mathrm{kDa}$ ) from spectrum Lab and Deionized water were used for purification of the synthesized compounds.

NMR spectra were recorded on a broker AMX 500 spectrometer and jeol ECP 500. Inverse gated ${ }^{13} \mathrm{C}$ NMR was performed on Brucker avance 400 and Brucker avance 500 spectrometers. For internal calibration tetramethylsilane was used at $12 \mathrm{MHz}$ with complete proton decoupling.

GPC measurements were achieved using an Agilent 1100 solvent delivery system with a manual injector, isopump and Agilent 1100 differential refractometer. The Brookhaven BIMWA7-angle light scattering detector was coupled to a size exclusion chromatography (SEC) to measure the molecular weight for each fraction of the polymer that was eluted from the SEC columns. For separation of the polymer samples, three $30 \mathrm{~cm}$ columns were used (10 $\mu \mathrm{m}$ PSS Suprema columns with pore sizes of $100 \AA$, $1000 \AA$, $3000 \AA$ ). Water was used as mobile 
phase; the flow rate was set at $1.0 \mathrm{~mL} \mathrm{~min}^{-1}$. All columns were held at room temperature. For each measurement, $100 \mu \mathrm{L}$ of samples with concentration of $5 \mathrm{mg} \mathrm{mL}^{-1}$ solution was injected. For acquisition of data from seven scattering angles (detectors) and differential refractometer Win GPC Unity from PSS was used. Molecular weight distributions were determined by comparison with pullulan standards (10 different sizes from 342 to $710000 \mathrm{~g} \mathrm{~mol}^{-1}$ ). Water was used as solvent with $0.1 \mathrm{M}$ $\mathrm{NaNO}_{3}$.

IR spectra were obtained with Nicolet AVATAR320FT-IR5SXC (Thermo Fisher Scientific, Waltham, MA, USA) with a DTGS detector from 4000 to $650 \mathrm{~cm}^{-1}$. Sample measurement was done by dropping a solution of compound and letting the solvent evaporate for a few seconds.

Matrix-Assisted Laser Desorption/Ionization Time-of-Flight Mass Spectroscopy (MALDI-TOF) was measured with a Bruker Ultraflex TOF/TOF in the positive ion mode using a linear pathway (LP). Alpha-cyano-4-hydroxycinnamic acid was used as matrix. The samples were prepared via the "dried-droplet method" by dropping $0.5 \mu \mathrm{L}$ of methanolic solution of the sample mixture and letting them dry in air. The mixture was prepared by mixing $100 \mu \mathrm{L}$ of a $2 \mathrm{mg} \mathrm{mL}^{-1}$ solution of the sample, $100 \mu \mathrm{L}$ of a saturated matrix solution, and $20 \mu \mathrm{L}$ of a terephthalic acid sodium salt solution (TA).

\section{Polymerization of glycidol}

$1.11 \mathrm{~g}(0.015 \mathrm{~mol})$ glycidol and $0.88 \mathrm{~g}(0.005 \mathrm{~mol})$ ascorbic acid were mixed in a polymerization ampule. Mixture was stirred under vacuum at different temperatures $\left(40{ }^{\circ} \mathrm{C}\right.$ and $\left.70{ }^{\circ} \mathrm{C}\right)$ to produce polyglycerols with different structural parameters. Reaction was terminated, when products with high viscosity were formed and the magnet bar did not rotate anymore. This reaction is repeated for $1.85 \mathrm{~g}(0.025 \mathrm{~mol})$ glycidol and $0.85 \mathrm{~g}$ $(0.005 \mathrm{~mol})$ ascorbic acid in temperature of $40{ }^{\circ} \mathrm{C}$. Afterwards, ampule contents were dissolved in water and dialyzed against the same solvent for $24 \mathrm{~h}$ via dialysis tube with molecular weight cut off $2000 \mathrm{~g} \mathrm{~mol}^{-1}$. The purified products were obtained after drying in a vacuum oven at $40{ }^{\circ} \mathrm{C}$ and reduced pressure.

\section{Results and discussion}

In order to synthesis polyglycerols free from traces of toxic agents, ascorbic acid was used as an activator for cationic ring opening polymerization of glycidol at ambient conditions (Scheme 1).

Different molar ratios of glycidol to ascorbic acid (G/As) and two different reaction temperatures were chosen to investigate the effect of these parameters on the structure of products (Table 1). Polyglycerols synthesized by G/As $=3 / 1$ molar ratio at $40{ }^{\circ} \mathrm{C}$ and $70{ }^{\circ} \mathrm{C}$ are abbreviated as $\mathrm{hPG}_{3 / 40}$ and $\mathrm{hPG}_{3 / 70}$, respectively. Also polyglycerol synthesized by G/As $=5 / 1$ molar ratio at $40{ }^{\circ} \mathrm{C}$ is abbreviated as $\mathrm{hPG}_{5 / 40}$. In the IR spectra, absorbance bands at $3300 \mathrm{~cm}^{-1}, 2891 \mathrm{~cm}^{-1}$ and $1100 \mathrm{~cm}^{-1}$ are corresponded to the hydroxyl, aliphatic $\mathrm{C}-\mathrm{H}$ and $\mathrm{C}-\mathrm{O}$ bonds of polyglycerols (Fig. 1). In ${ }^{1} \mathrm{H}$ NMR spectra, signals at 3.3-4.2 ppm are assigned to the protons of hPGs (Fig. 2) due to the fact that both IR and ${ }^{1} \mathrm{H}$
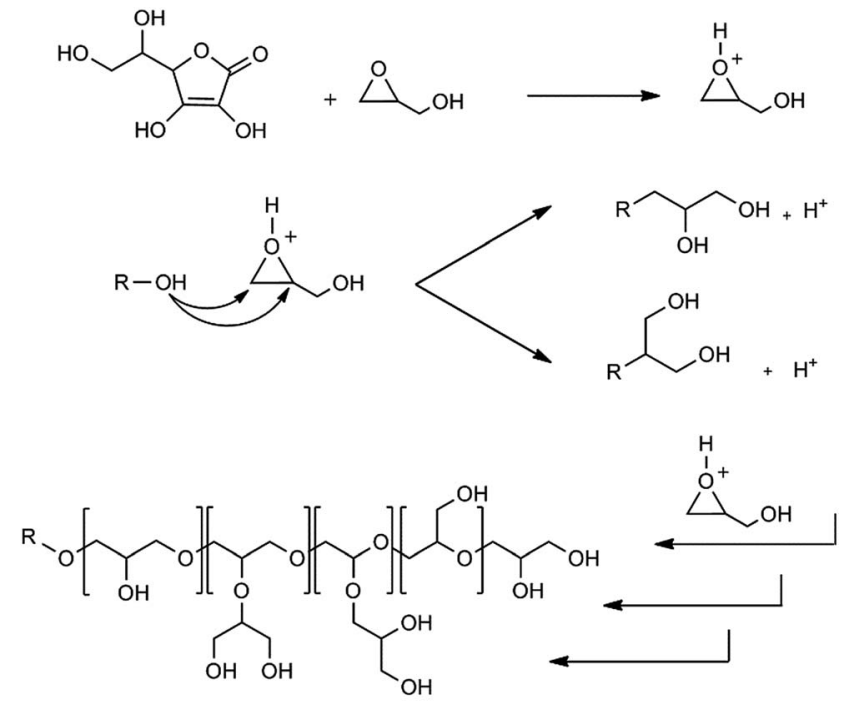

$\mathrm{ROH}$ is the monomer or a polymer chain

Scheme 1 Schematic representation of the proposed mechanism for the ring opening polymerization of glycidol by ascorbic acid.

Table 1 Degree of branching, molecular weight, reactions time, chemical shift and relative abundance of the linear, terminal, and dendritic units of the hPGs synthesized by different G/As molar ratios at different temperatures

\begin{tabular}{lllll}
\hline Structural units & Shift (ppm) & $\mathrm{hPG}_{3 / 70}$ & $\mathrm{hPG}_{5 / 40}$ & $\mathrm{hPG}_{3 / 40}$ \\
\hline $\mathrm{L}_{1,3}$ & $60.4-61.1$ & 0.70 & 0.65 & 0.59 \\
$2 \mathrm{~T}_{2}$ & $61.1-61.7$ & 0.30 & 0.32 & 0.41 \\
$\mathrm{~T}_{1}$ & $62.4-63$ & 0.49 & 0.46 & 0.36 \\
$\mathrm{~L}_{1,4}, \mathrm{~L}_{1,3}$ & $68.6-69.9$ & 1.13 & 1.08 & 1.13 \\
$2 \mathrm{D}, 2 \mathrm{~T}$ & $70-71.6$ & 2.47 & 2.27 & 1.63 \\
$2 \mathrm{~L}_{1,4}$ & $71.8-72.8$ & 1.35 & 1.48 & 1.12 \\
$\mathrm{D}$ & $77.7-78.9$ & 0.57 & 0.53 & 0.65 \\
$\mathrm{~L}_{1,3}$ & $79.3-80$ & 0.38 & 0.47 & 0.41 \\
$\mathrm{~T}_{2}$ & $81-81.5$ & 0.13 & 0.12 & 0.22 \\
Reaction time (h) & & 3 & 16 & 16 \\
Degree of branching & & 0.45 & 0.43 & 0.53 \\
Linear 1, 3 units (\%) & & 28 & 26 & 27 \\
Linear 1, 4 units (\%) & & 27 & 30 & 26 \\
Dendritic units (\%) & & 23 & 21 & 30 \\
Terminal units (\%) & & 22 & 23 & 17 \\
$M_{\mathrm{n}}(\mathrm{GPC})\left(\mathrm{g}\right.$ mol $^{-1}$ ) & & 2516 & 2256 & 2138 \\
$M_{\mathrm{w}}(\mathrm{GPC})\left(\mathrm{g} \mathrm{mol}^{-1}\right.$ ) & & 4417 & 3942 & 3334 \\
PDI & & 1.75 & 1.75 & 1.56 \\
& & & &
\end{tabular}

NMR spectra do not show absorbance bands and signals of ascorbic acid, this compound plays only the role of activating agent and it is not incorporated in the structure of polyglycerols.

Mechanism of polymerization and the effect of G/As molar ratio as well as reaction temperature on the structure of the synthesized polymers was investigated by inverse gated ${ }^{13} \mathrm{C} N M R$ (Fig. 3). According to these spectra and using equation presented by Frey et al. ${ }^{13,26}$ relative abundance of structural unites as well as degree of branching (DB) of hPGs were determined (Table 1). In comparison to the anionic ring opening polymerizations, DB of 


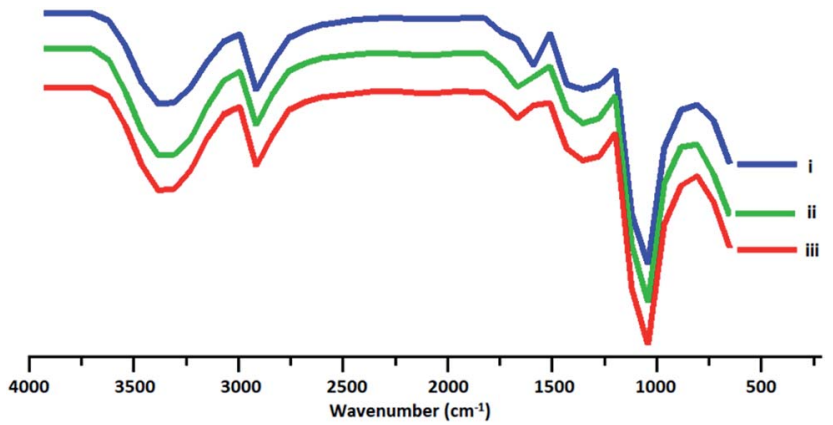

Fig. 1 IR spectra of the synthesized polyglycerols. (i, ii and iii) Spectra's are belong to $\mathrm{hPG}_{3 / 40}$ and $\mathrm{hPG}_{3 / 70}$ and $\mathrm{hPG}_{5 / 40}$, respectively.

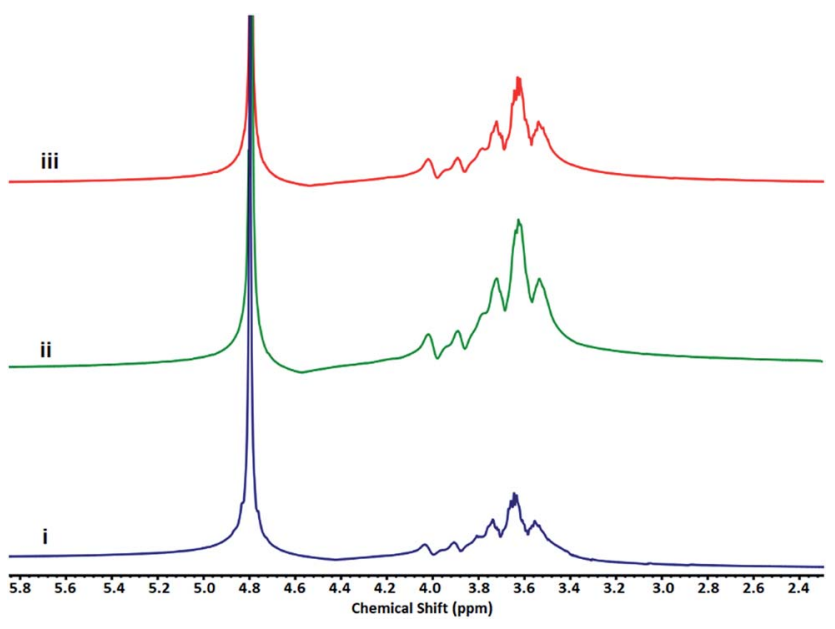

Fig. $2{ }^{1} \mathrm{H}$ NMR spectra of hPGs. (i, ii and iii) Spectra's are belong to $h \mathrm{PG}_{3 / 40}$ and $\mathrm{hPG} / 70$ and $\mathrm{hPG} 5 / 40$, respectively.

polymers is in the range of $0.44-0.53$. However, it is higher than the reported DB for the cationic ring opening polymerization of glycidol. ${ }^{23,24}$ This is due to the higher relative abundance of $\mathrm{L}_{1,4}$ structural unites (Table 1). Moreover, the relative abundance of the dendritic blocks (D\%) and therefore DB of hPGs depend on the G/As molar ratios and temperature, inversely.

Inverse gated ${ }^{13} \mathrm{C}$ NMR spectra of the synthesized polyglycerols show that polymers are containing $\mathrm{L}_{1,4}$ units in addition to $\mathrm{L}_{1,3}$ units (Fig. 3 and Table 1).

It is known that activated chain end (ACE) mechanism results in the $L_{1,3}$ units, exclusively. Therefore, presence of $L_{1,4}$ units in the structure of the synthesized hPGs confirm that glycidol is polymerized by ascorbic acid through the activated monomer (AM) mechanism. ${ }^{16,23,27}$ Accordingly, the proposed mechanism for the polymerization of glycidol by ascorbic acid is as below:

(i) Protonation of glycidol.

(ii) Polymerization of protonated monomers by glycidol.

Different groups in the structure of polyglycerol were characterized by DEPT $-{ }^{13}$ CNMR (Fig. 4). Clearly, all well-known signals for polyglycerols are appeared in this spectra. According to gel permeation chromatography (GPC) the synthesized polyglycerols showed molecular weights in the range of 2138-
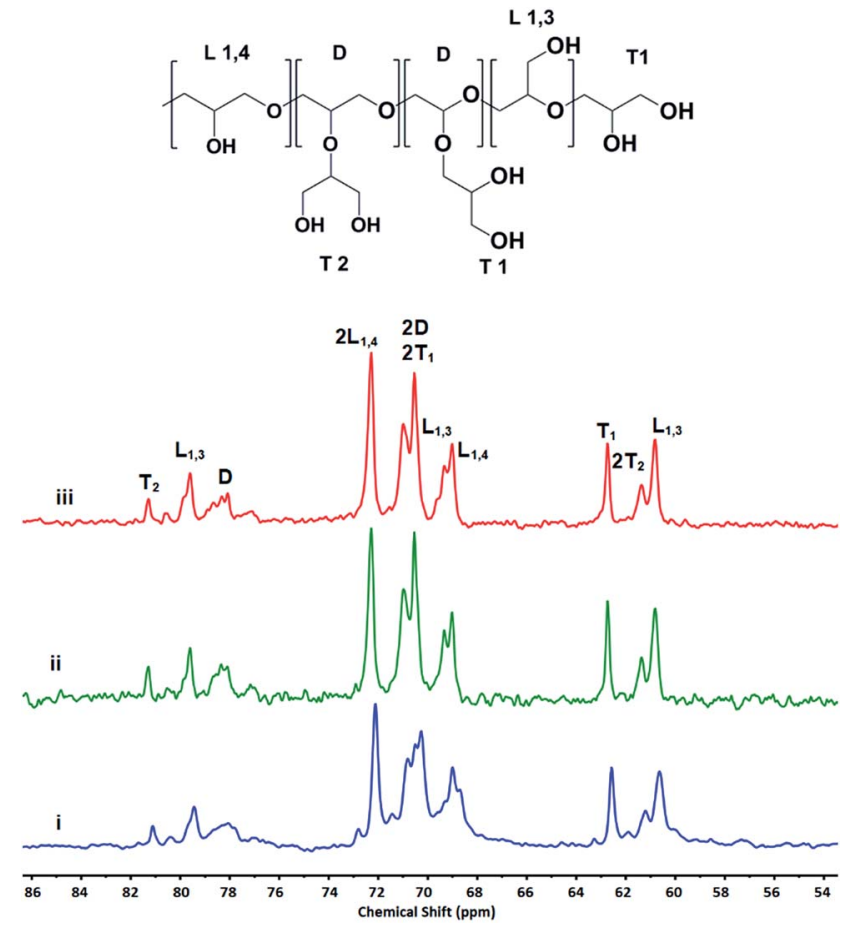

Fig. 3 Assignment of structural units of synthesized hPGs by different $\mathrm{G} /$ As ratios at $40{ }^{\circ} \mathrm{C}$ and $70{ }^{\circ} \mathrm{C}$ in the inverse gated ${ }^{13} \mathrm{C}$ NMR spectra.

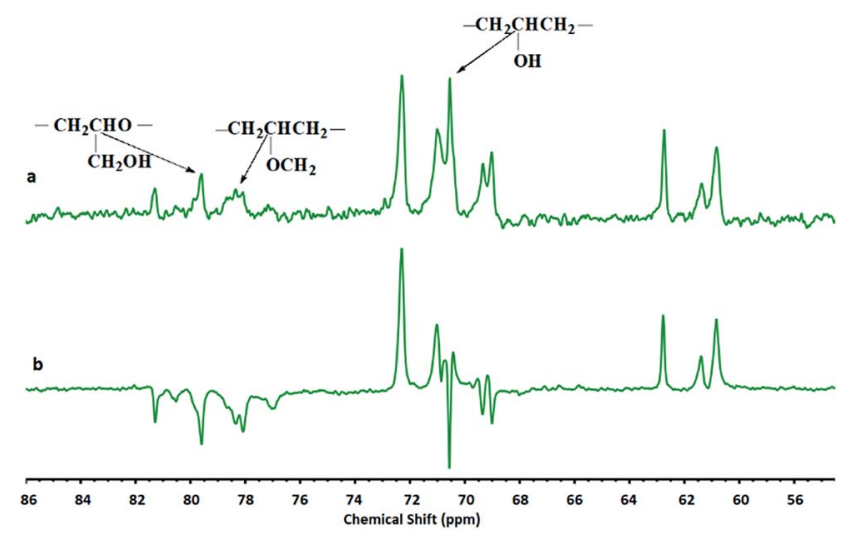

Fig. 4 (a) Inverse gated ${ }^{13} \mathrm{C} N M R$ of $\mathrm{hPG}_{3 / 70}$ and (b) ${ }^{13} \mathrm{C}$ DEPT spectrum of $\mathrm{hPG}_{3 / 70}$.

$2516 \mathrm{~g} \mathrm{~mol}^{-1}$. It was found that increasing the temperature of polymerization increase the molecular weight of the synthesized polyglycerols, while G/As molar ratio do not affect it significantly (Table 1).

MALDI-TOF MS spectrometry show that the molecular mass of the synthesized polymers is a multiple of 74 which is molecular mass of glycidol. In other words the mass distribution of the hPGs follow out 74n, where $n$ is the number of repeating units in polymer molecule (Fig. 6-8). The other mass distribution is due to hydrated polymer as it has 18 units ( $m_{\mathrm{w}}$ of $\mathrm{H}_{2} \mathrm{O}$ ) difference with the main polymer mass.

This result show that ascorbic acid is not incorporated in the structure of the synthesized polyglycerols. 


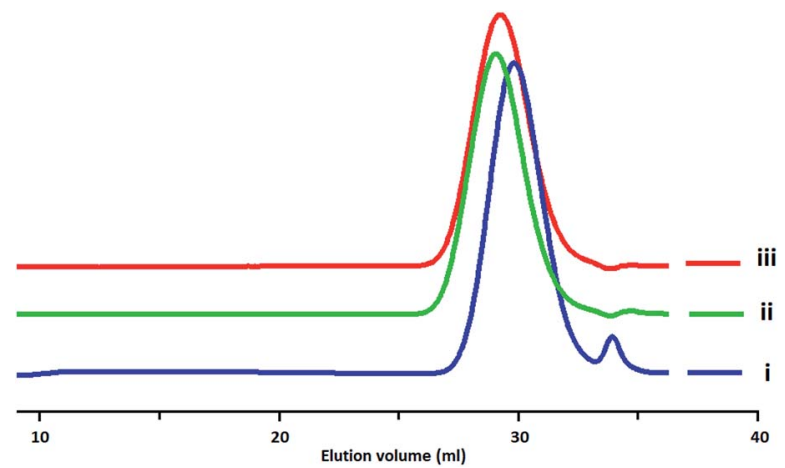

Fig. 5 GPC diagrams of hPGs.

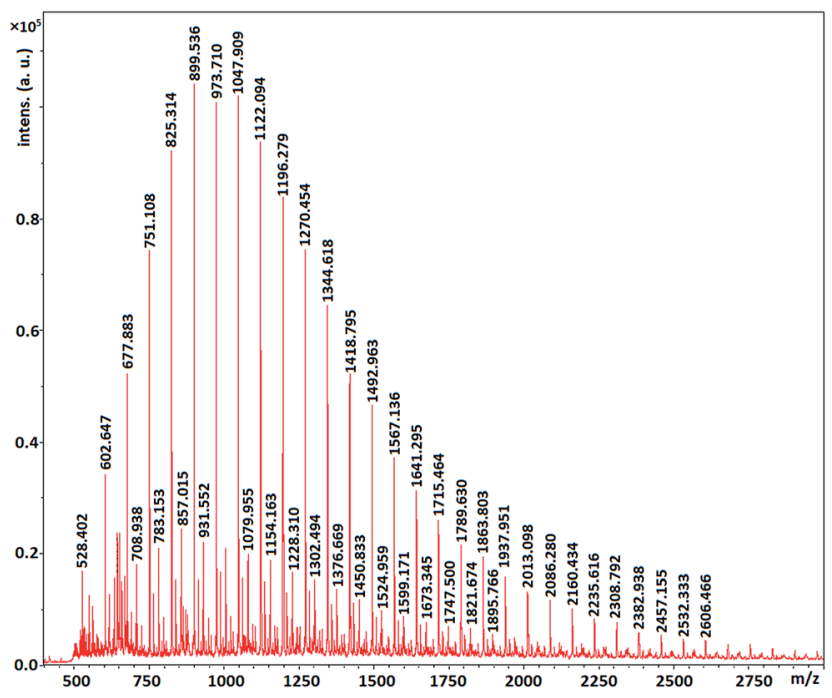

Fig. 6 MALDI-TOF mass spectra of $\mathrm{hPG}_{3 / 40}$.

Polydispersity index (PDI) of the synthesized polymers was specified by gel permeation chromatography (GPC) (Fig. 5). It was found that PDI amounts are depended on the G/As molar

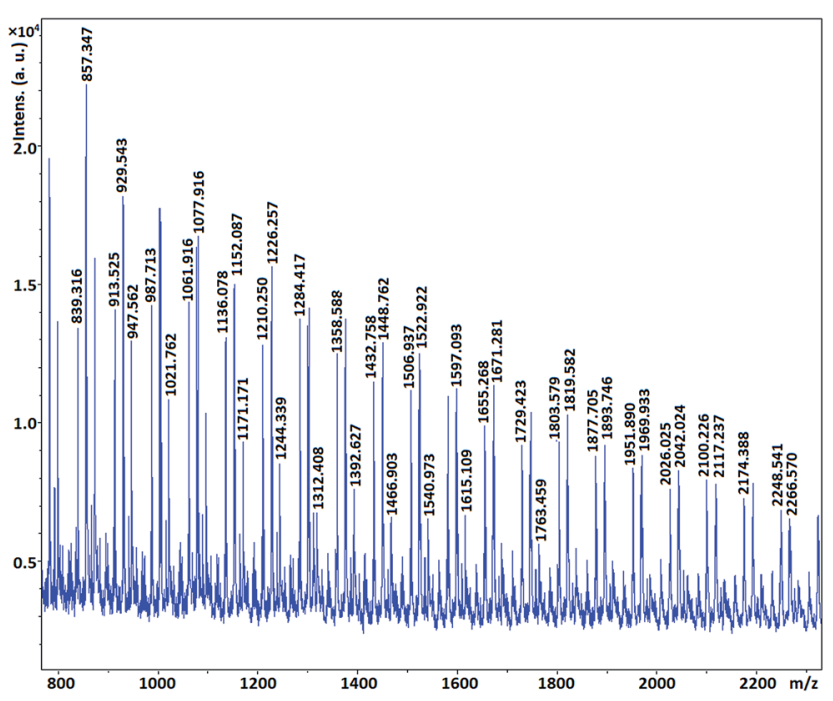

Fig. 7 MALDI-TOF mass spectra of $\mathrm{hPG}_{3 / 70}$.

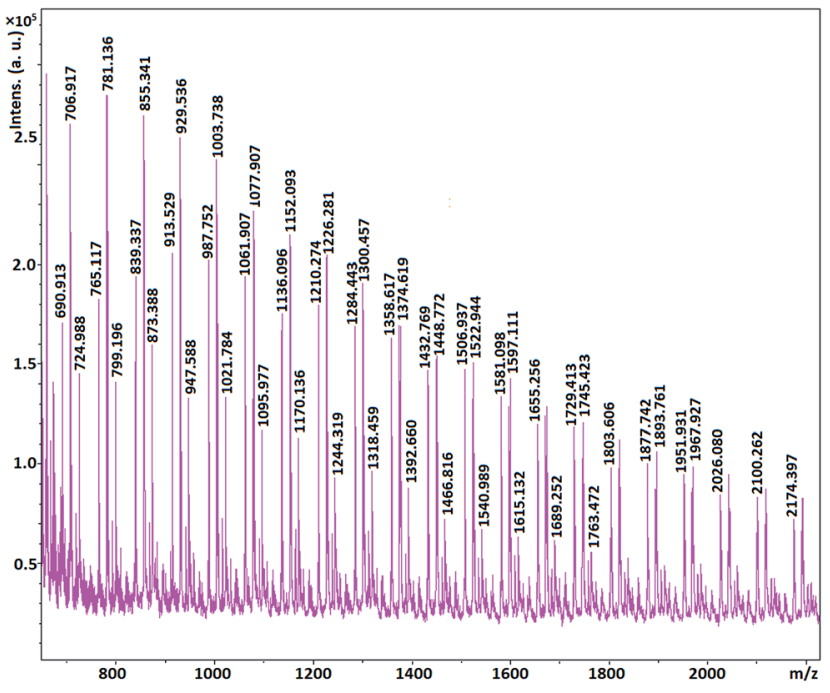

Fig. 8 MALDI-TOF mass spectra of $\mathrm{hPG}_{5 / 40}$.

ratios. The greater amount of molar ratio and upper temperature resulted in the higher PDI (Table 1).

According to the data in Table 1, while changing the reaction temperature and molar ratio do not show a big effect on the relative abundance of linear units, they highly change that of dendritic units. At the same temperature the higher molar ratio of ascorbic acid would result in the higher degree of branching. Since the higher amount of ascorbic acid protonate the more glycidol monomers and the chance for ring opening reaction of both hydroxyl groups would increases and result in high DB.

\section{Conclusions}

In summary, a new method for the preparation of polyglycerols using ascorbic acid as a biocompatible compound is reported. In this method, cationic ring opening polymerization of glycidol by ascorbic acid through the activated monomer (AM) mechanism results in polyglycerols with the degree of branching in the range of $0.44-0.53$. The synthesized polyglycerols by this method could be used for different biomedical applications, due to green synthesis procedure.

\section{Conflicts of interest}

There are no conflicts of interest between authors.

\section{Acknowledgements}

Authors would like to thanks Prof. Rainer Haag for his supports.

\section{Notes and references}

1 A. Brändle and A. Khan, Polym. Chem., 2012, 3, 3224.

2 Y. Deng, J. K. Saucier-Sawyer, C. J. Hoimes, J. Zhang, Y.-E. Seo, J. W. Andrejecsk and W. M. Saltzman, Biomaterials, 2014, 35, 6595. 
3 Z. Xu, Y. Zhang, Q. Hu, Q. Tang, J. Xu, J. Wu, T. B. Kirk, D. Ma and W. Xue, Mater. Sci. Eng. Carbon, 2017, 71, 965.

4 E. Mohammadifar, F. Daneshnia, M. Kehtari, A. N. Kharat and M. Adeli, Polym. Int., 2016, 65, 559.

5 N. Cengiz, J. Rao, A. Sanyal and A. Khan, Chem. Commun., 2013, 49, 11191.

6 A. Saha, S. De, M. C. Stuparu and A. Khan, J. Am. Chem. Soc., 2007, 134, 17291.

7 M. Imran ul-haq, B. F. L. Lai, R. Chapanian and J. N. Kizhakkedathu, Biomaterials, 2012, 33, 9135.

8 S. Gupta, R. Tyagi, V. S. Parmar, S. K. Sharma and R. Haag, Polymer, 2012, 53, 3053.

9 M. Calderón, M. A. Quadir, S. K. Sharma and R. Haag, Adv. Mater., 2010, 22, 190.

10 A. L. Sisson and R. Haag, Soft Matter, 2010, 6, 4968.

11 H.-Q. Wu and C.-C. Wang, Langmuir, 2016, 32, 6211.

12 S. R. Sandler and F. R. Berg, J. Polym. Sci., Polym. Chem. Ed., 1966, 4, 1253.

13 A. Thomas, S. S. Müller and H. Frey, Biomacromolecules, 2014, 15, 1935.

14 S. Binder, I. Gadwal, A. Bielmann and A. Khan, J. Polym. Sci., Part A: Polym. Chem., 2014, 52, 2040.

15 S. Lee, D. H. Kim, D. H. Kang, B.-S. Kim, K. Saito, S. Sasaki, Y. Oishi and Y. Shibasaki, Biomacromolecules, 2013, 14, 2171.
16 E. Mohammadifar, A. Nemati Kharat and M. Adeli, J. Mater. Chem. B, 2015, 3, 3896.

17 A. Dworak, W. Walach and B. Trzebicka, Macromol. Chem. Phys., 1995, 196, 1963.

18 C. Schüll and H. Frey, ACS Macro Lett., 2012, 1, 461.

19 D. Wilms, S.-E. Stiriba and H. Frey, Acc. Chem. Res., 2010, 43, 129.

20 I. Gadwal, S. Binder, M. C. Stuparu and A. Khan, Macromolecules, 2014, 47, 5070.

21 A. Timothy Royappa, M. L. Vogt and V. Sharma, J. Appl. Polym. Sci., 2004, 91, 1344.

22 T. V. Grinevich, N. G. Taganov, M. L. Pridatchenko and A. A. Berlin, Dokl. Chem., 2017, 472, 1.

23 K. Petrak, Bull. Mater. Sci., 1989, $12,41$.

24 K. Petrak, Drug Discovery Today, 2005, 10, 1667.

25 B. R. Spears, M. A. Marin, J. R. Montenegro-Burke, B. C. Evans, J. McLean and E. Harth, Macromolecules, 2016, 49, 2022.

26 B. R. Spears, J. Waksal, C. McQuade, L. Lanier and E. Harth, Chem. Commun., 2013, 49, 2394.

27 E. Mohammadifar, A. Bodaghi, A. Dadkhahtehrani, A. Nemati Kharat, M. Adeli and R. Haag, ACS Macro Lett., 2017, 6, 35 . 